\title{
Integration und Abgrenzung. Vorüberlegungen zu den politischen Funktionen überregionaler Heiligtümer in der griechischen Staatenwelt*
}

\author{
Peter Funke
}

Das Spannungsgefüge zwischen Religion und Politik wird derzeit an der Westfälischen Wilhelms-Universität Münster im Exzellenzcluster „Religion und Politik in den Kulturen der Vormoderne und Moderne" in einem sehr weiten Rahmen thematisiert und von Forscherinnen und Forschern aus fast allen Bereichen der Theologischen, Juristischen und Philosophischen Fakultäten sowohl in synchronen Querschnitts- wie auch in diachronen Längsschnittanalysen untersucht. ${ }^{1}$ In diesem Zusammenhang steht auch das von mir geleitete Teilprojekt, in dem der Frage nachgegangen wird, welche politische Rolle Religion und Kult im komplexen zwischenstaatlichen Miteinander der antiken griechischen Poliswelt gespielt haben. Nun ist auch dies noch ein sehr weites Themenfeld, innerhalb dessen vorerst zwei Aspekte im Vordergrund stehen: Einerseits geht es um die Bestimmung des Stellenwertes von Religion und Kult in zwischenstaatlichen Vereinbarungen - Bündnisverträgen, Friedenschlüssen, Asylieverträgen etc. - und andererseits um die Analyse der politischen Funktionen von so genannten ,überregionalen“ Heiligtümern. Auch wenn der erstgenannte Aspekt im Folgenden weitgehend außer Betracht bleiben wird $^{2}$ und

* In den folgenden Darlegungen möchte ich einige Grundüberlegungen zur Diskussion stellen, die ich erstmals im Rahmen des Münsteraner DFG-Sonderforschungsbereiches 493 „Funktionen von Religionen in antiken Gesellschaften“ entwickelt hatte und die ich jetzt im Münsteraner Exzellenzcluster „Religion und Politik in den Kulturen der Vormoderne und Moderne" weiter bearbeiten kann. Der Text geht zurück auf einen Vortrag, den ich im Januar 2009 in Kairo auf einem Arbeitstreffen des Forschungsclusters 4 „Heiligtümer" des Deutschen Archäologischen Instituts gehalten habe. Ich danke allen Diskutanten für die anregenden und ertragreichen Wortbeiträge. - Die bibliographischen Angaben sind auf ein Minimum reduziert und verweisen vielfach auf weiterführende Literatur, um ein Überborden der Anmerkungen zu vermeiden, zumal es hier zunächst einmal nur um einige grundlegende Vorüberlegungen geht.

1 Zu den Zielsetzungen und Themenfeldern des Münsteraner Exzellenzclusters „Religion und Politik in den Kulturen der Vormoderne und Moderne“ vgl. die entsprechende Website: http://www.uni-muenster.de/Religion-und-Politik/.

2 Mit diesem Bereich beschäftigen sich innerhalb des Teilprojektes zwei Promotionsvorhaben: Sebastian Scharff untersucht unter dem Arbeitstitel „Parteiische Götter - konkurrierende Götter" die Rolle von Kulten und Heiligtümern in antiken Staatsverträgen, 
nur der zweite Aspekt näher ausgeführt werden soll, können die hier in einem ersten Teil vorangestellten Grundüberlegungen doch eine gemeinsame Basis für die genannten Untersuchungen bilden, da beide Bereiche in mancherlei Weise ursächlich miteinander verbunden sind. Den anschließenden Ausführungen eignet in Vielem noch ein vorläufiger Charakter und sie können daher kaum mehr sein als die Markierung eines Ausgangspunktes für weitere Untersuchungen.

In der antiken Staatenwelt - vor allem der griechischen - , die in ihrem Denken und Handeln unabänderlich orientiert blieb an den Maximen von Ehre, Rache und Vergeltung, die auch Vergebung miteinschließen konnten, war Gewalttätigkeit allgegenwärtig; Kriege und Bürgerkriege in ihrer brutalsten Ausprägung bildeten eher die Regel als die Ausnahme. ${ }^{3}$ Sowohl der innerstaatliche Zusammenhalt in den einzelnen griechischen Poleis wie auch das zwischenstaatliche Beziehungsgeflecht befanden sich permanent in einem prekären Zustand, dessen Stabilisierung nur auf dem Wege gemeinschaftlicher Vereinbarungen zu erreichen war. Die Sicherung des Zusammenhalts der bürgerlichen Gemeinschaften in den Poleis und die Gewährleistung eines regulierten zwischenstaatlichen Miteinanders dieser Gemeinschaften untereinander wie auch mit nichtgriechischen Staaten erforderten die Aufstellung und Durchsetzung von Verhaltensnormen, die auf die Einhegung und Kontrolle von Gewalt hinwirken sollten, wenn sie denn schon nicht gänzlich verhindert werden konnte. Voraussetzung für die Formulierung und die Anwendung solcher Normen war zunächst einmal deren Akzeptanz bei allen jeweils Beteiligten. Die bloße gegenseitige Akzeptanz allein wurde aber in der Regel nicht als ausreichend anerkannt; es bedurfte offenbar vielmehr der Verankerung der Normen in einem kollektiven Sinnzusammenhang. Diesen vermochten in besonderer Weise Religion und Kult zu bieten, da diese sowohl auf der Ebene der Einzelstaaten - der Poleis und Stämme - wie auch auf gesamtgriechischer Ebene zentrale, identitätsstiftende bzw. identitätswahrende Funktionen erfüllten oder doch zumindest erfüllen konnten und daher für einen begründenden und absichernden Rekurs nicht nur geeignet, sondern sogar unabdingbar waren. Darüber hinaus konnten Religion und Kult offenbar sogar auch zwischen griechischen und nichtgriechischen Entitäten Grundlagen für eine gemeinsame

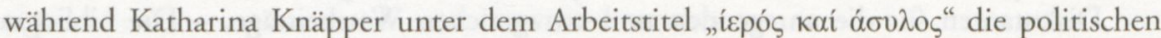
und religiösen Funktionen hellenistischer Asylieverleihungen analysiert.

3 Grundlegend dazu H.-J. Gehrke, Stasis- Untersuchungen zu den inneren Kriegen in den griechischen Staaten des 5. und 4. Jhdt. v. Chr. (1985); ders., „Die Griechen und die Rache: Ein Versuch in historischer Psychologie“, Saeculum 38 (1987) 121-149; vgl. darüber hinaus (mit der neueren Literatur) H. van Wees (Hg.), War and Violence in Ancient Greece (2000); G. Fischer - S. Moraw (Hgg.), Die andere Seite der Klassik. Gewalt im 5. und 4. Jahrhundert v. Chr. (2005); B. Seidensticker - M. Vöhler (Hgg.), Gewalt und Formen ihrer ästhetischen Repräsentation im 5. Jahrhundert v. Chr. (2006); Martin Zimmermann (Hg.), Extreme Formen von Gewalt in Bild und Text des Altertums (2009). 
Vertrauensbasis schaffen, so dass etwa die Beeidung zwischenstaatlicher Vereinbarungen durch die Berufung auf die je eigenen Götter gegenseitig akzeptiert wurde.

Allerdings war ein solcher Rekurs nicht nur, aber vor allem immer dann, wenn der Bereich der Polisreligion überschritten wurde, in überaus komplexe Rahmenbedingungen eingebunden: Die polytheistische Religion der Griechen kannte zwar ein gesamtgriechisches Pantheon und die Griechen waren sich durchaus einer im Grunde gemeinsamen Religion bewusst und praktizierten diese auch etwa an den panhellenischen Kult- und Orakelstätten; es gab aber dennoch eben auch eine Konkurrenz der Polisreligionen und es gab die parteiischen Götter, die sich etwa im Kampf um Troja zerstritten und die sich unversöhnlich und rachsüchtig auf die eine oder andere der gegnerischen Seiten stellten. Und noch komplexer wurden die Gegebenheiten, wenn es in politicis zum Beispiel bei vertraglichen Vereinbarungen darum ging, einen gemeinsamen Bezugsrahmen in religiosis zu finden, dieser aber im Verhältnis zu fremden, nicht-griechischen Religionen auszumachen war. Es ist bemerkenswert, dass selbst in solchen Fällen in der Regel eine gemeinsame Verständigungsebene herzustellen war.

Im Folgenden soll der Blick aber zunächst nur auf die griechische Staatenwelt fokussiert bleiben. Religion und Kult bildeten hier offenbar einen bestimmenden Faktor eines im Vorangegangenen nur knapp skizzierten Akzeptanzsystems, dessen Grundlagen, Funktionsmechanismen und Wirkungsweisen unter religionsgeschichtlicher Perspektive immer noch einer genaueren Analyse bedürfen. Der Rekurs auf die religiöse Sphäre gestaltete sich vergleichsweise problemlos, da die polytheistische Welt in ihrer Wesenhaftigkeit Bestandteil eines allseitig anerkannten religiösen Gesamtsystems war, also eben nicht schon gleichzeitig religiöse Pluralität bedeutete, sondern auf einem fest gefügten, allseits akzeptierten Pantheon gründete und damit dann doch wieder eine gewisse religiöse und kultische Einheit implizierte. ${ }^{4}$

Hier aber liegt auch eine erste große Schwierigkeit: Wie einheitsstiftend war eigentlich dieses religiöse Zusammengehörigkeitsgefühl bei den Griechen. Bekanntlich existierten die (antiken) Griechen als eine politische Entität nicht; vielmehr waren sie in mehr als achthundert politischen Einheiten ganz unterschiedlichen Zuschnitts - als Polis oder Stammesverbund - organisiert, von denen eine jede auf die unbedingte Wahrung ihrer Autonomie und Freiheit

$4 \mathrm{Zu}$ diesem der griechischen und auch römischen Religion inhärenten Spannungsgefüge vgl. die grundlegende Studie von H. S. Versnel, Ter Unus: Isis, Dionysos, Hermes: Three studies in Henotheism (= Inconsistencies in Greek and Roman religion I) (1990); s. auch (mit weiterer Literatur) B. Gladigow, „Polytheismus“, Handbuch religionswissenschaftlicher Grundbegriffe 4 (1998) 321-330 und die entsprechenden Beiträge in T. Schabert M. Riedl (Hgg.), Gott oder Götter? God or Gods? (2008). 
bedacht war. Dieser politischen Vielfalt, die wir uns nicht bunt genug vorstellen können, stand eine kulturelles Zusammengehörigkeitsgefühl gegenüber, das in dem Bewusstsein von der Existenz eines alle Griechen gemeinsamen "Hellenikón“ zum Ausdruck kam, zu dessen zentralen Bestandteilen gerade auch die gemeinsame Religion gehörte, was später noch näher auszuführen ist. Gleichwohl blieb ein permanentes Spannungsgefüge zwischen den polisbezogenen und den panhellenischen Dimensionen griechischer Religion, das die Rahmenbedingungen der politischen Funktionen überregionaler Heiligtümer grundlegend und voraussetzungsreich bestimmte und auf das ich daher zunächst näher eingehen möchte.

Von der griechischen Religion sprechen heißt immer zugleich auch von der Polis sprechen. „The polis provided the fundamental framework in which Greek religion operated ... The Greek polis articulated religion and was itself articulated by it; religion became the polis' central ideology. ${ }^{\text {"5 }}$ Mit dieser Feststellung hat Christiane Sourvinou-Inwood den Grundzug griechischer Religion klar gekennzeichnet. Die griechische Religion ist primär Polis-Religion. Religion und Kult waren daher ein unmittelbares Abbild des politischen Systems der griechischen Poliswelt und damit in ihren Erscheinungsformen durch eine entsprechende Vielfalt gekennzeichnet. Jede Polis verfügte bekanntlich über eigene zentrale Heiligtümer, Festtage, Kultkalender und Jahresrhythmen, die ein entscheidendes Bindeglied für den Zusammenhalt des jeweiligen Bürgerverbandes bildeten. Sie waren fester Bestandteil der Polisidentität, die in entscheidender Weise religiös begründet wurde. Es sei hier nur auf die Untersuchungen von François de Polignac über „Kulte, Territorium and die Ursprünge der griechischen Polis" verwiesen, der mit Blick auf die Heiligtümer in den griechischen Poleis von einer religiösen Bipolarität griechischer Städte spricht und damit eine doppelte Funktion der Kultstätten in den Poleis beschreibt: Einerseits die Konstituierung eines sozialen Raumes innerhalb einer Polis (also in Bezug auf den Bürgerverband und teilweise durchaus auch die übrigen Bewohner), zugleich aber auch durch die Errichtung von Grenzheiligtümern die Fixierung des Polisterritoriums und damit die Abgrenzung zu den umliegenden, auf gleiche Weise festgelegten Polisterritorien. ${ }^{6}$

Was de Polignac in seinem Buch entwickelt, findet schon im sechsten Buch der platonischen Nomoi eine Entsprechung, wo nach langen Ausführungen über die beste Form des Staates auch die Frage der baulichen Gestaltung aufgeworfen wird: „Und da unser Staat ja neu und bisher noch unbewohnt ist, so muss er sich natürlich so ziemlich um das gesamte Bauwesen kümmern und überlegen, wie er es mit allen Einzelheiten und so auch mit den Heiligtümern und Mauern

5 Chr. Sourvinou-Inwood, „What is Polis Religion?", in: R. Buxton (Hg.), Oxford Readings in Greek Religion (2000) 13.

6 F. de Polignac, Cults, Territory, and the Origins of the Greek City-State (1995). 
halten soll." Und als erstes wird dann der Bau der Heiligtümer beschrieben: „Die Heiligtümer soll man rings um den ganzen Markt und rings um die ganzen Stadt im Kreis an hochgelegenen Plätzen erbauen. "7 Die Heiligtümer um den Markt als Integrationsfaktor und die Heiligtümer auf den Höhen rings um die Polis als Abgrenzungsfaktor: Prägnanter lässt sich die von de Polignac bezeichnete religiöse Bipolarität und die doppelte Funktion der Kultstätten in den Poleis kaum beschreiben.

Wenn nun aber der "Polis-Religion" ein so hoher Stellenwert zukam, ergibt sich aber zugleich auch die Frage nach der Exklusivität aller dieser Polis-Religionen. Was bedeutet „Polis-Religion“ hinsichtlich der Teilhabe an allen diesen Heiligtümern und Kulten? Eine Antwort auf diese Frage kann sich nicht an den zahllosen Vorschriften und Reglements orientieren, durch die bestimmten Gruppen oder Individuen aufgrund spezifischer Begründungen der Zugang zu einzelnen Heiligtümern und Kulten verwehrt wurde. Ich erinnere - um nur einige wenige Exempel anzuführen - an den Ausschluss der Thebaner von der Befragung des Orakels im Amphiareion ${ }^{8}$ oder das Verbot für Dorer, den Tempel der Athena auf der Athener Akropolis zu betreten. ${ }^{9}$ Es gab Heiligtümer und Kulte, die nur Frauen oder Männern offen standen; andere Heiligtümer wiederum durften nur von Priestern oder Kultbeamten betreten werden und wieder andere Heiligtümer waren überhaupt unbetretbar (abaton). Diese Aufzählung ließe sich noch um Vieles erweitern. ${ }^{10}$

Solche Tabuisierungen fanden ihre Begründung im jeweiligen Einzelfall und sind daher wenig geeignet, um auf unsere Frage nach der Exklusivität von PolisReligion eine angemessene Antwort zu finden. Hier gilt es vielmehr nach einem spezifischen Tabu zu fragen, das die Zugehörigkeit zur Polis zugleich auch zum Kriterium macht für den Zugang zu Heiligtümern und für die Beteiligung an Kulten. In den Quellen finden sich diesbezüglich erstaunlich wenig eindeutige Aussagen wie etwa die gleichlautenden Inschriften auf zwei Architravblöcken in Delos, die dem so genannten Archegesion, dem Heiligtum des mythischen Archegeten von Delos, Anios, zuzuweisen sind. In strikter Form wird dort

7 Platon, Nomoi 778b-c (Übersetzung nach K. Schöpsdau).

8 Herodot 8,134,2.

9 Herodot 5,72,3-4; vgl. dazu R. Parker, Cleomenes on the Acropolis. An Inaugural Lecture delivered before the University of Oxford on 12 May 1997 (1998).

10 S. etwa die Zusammenstellung bei M. P. Nilsson, Geschichte der griechischen Religion, Bd. I $\left(1957^{3}\right)$ 75-77; vgl. auch S. Krauter, Bürgerrecht und Kultteilnahme: Politische und kultische Rechte und Pflichten in griechischen Poleis, Rom und antikem Judentum (2004) bes. 53-113; P. Funke, „Fremde und Nichtbürger in den griechischen Heiligtümern der antiken Mittelmeerwelt. Eine historische Einführung", in: A. Naso (Hg.), Stranieri e non cittadini nei santuari greci (2006) 1-12. 


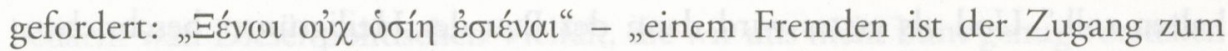
Heiligtum verboten". 11

Eine solche rigorose Ausgrenzung von xenoi findet sich allerdings nur selten und scheint eher die Ausnahme gewesen zu sein. ${ }^{12}$ Das dürfte seinen Grund darin haben, dass die Poliskulte in der Regel durchaus von einer gewissen Offenheit geprägt waren. Diese Offenheit der Polis-Kulte bedeutete allerdings nicht, dass Bürger und xenoi in gleicher Weise an den Kulten partizipierten, es sei denn, dass es besondere Gründe gab, auch die xenoi stärker an den Kulten zu beteiligen, um sie auf diese Weise auch enger an die Polis zu binden. Darauf zielten beispielsweise die Bemühungen der Athener ab, die Mitglieder des Delisch-Attischen Seebundes - teilweise sogar unter Zwang - an den Festfeiern der Panathenäen $\mathrm{zu}$ beteiligen. Den Athenern ging es eben gerade darum, das Fremdsein der Bündner wenn schon nicht im rechtlichen Sinne, so doch zumindest über Kult und Religion zu überwinden und auf diese Weise das Gefüge ihres Bündnissystems zu festigen. ${ }^{13}$ In der Regel galten für Fremde aber doch besondere Regeln, sofern sie nicht grundsätzlich von den Kulten ausgeschlossen waren. In dem Gesetz, das die Ostlokrer als Grundlage für ihre Koloniegründung in Naupaktos beschlossen, wurde ausdrücklich festgelegt, dass die Lokrer, die sich in Naupaktos ansiedeln, da sie dann ja Naupaktier seien, in ihrer ursprünglichen Heimat Ost-Lokris nur als xenoi an heiligen Handlungen teilnehmen und opfern dürften. ${ }^{14}$ Gerade dieses Beispiel einer Koloniegründung zeigt besonders deutlich, wie ausgeprägt der Bezug des Bürgers zu seiner Polis

11 Inscriptions de Délos 68; s. auch B. Le Guen-Pollet, La vie réligieuse dans le monde grec du Ve au IIIe siècle avant notre ère: Choix de documents épigraphiques traduits et commentés (1991) Nr. 22; R. Körner, Inschriftliche Gesetzestexte der frühen griechischen Polis. Aus dem Nachlaß von R. Körner hg. von K. Hallof (1993) Nr. 54. Diese Inschrift wurde als Inscriptions de Délos 68A gemeinsam mit der neu entdeckten, gleichlautenden Inschrift Inscriptions de Délos $68 \mathrm{~B}$ von P. A. Butz, „The double publication of a sacred prohibition on Delos: ID 68, A and B“, Bulletin de correspondance hellénique 118 (1994) 69-98 veröffentlicht; vgl. Supplementum Epigraphicum Graecum 44, 678.

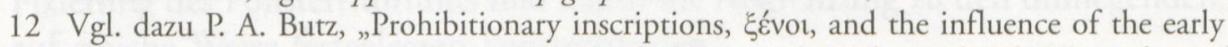
Greek polis“, in: R. Hägg (Hg.), The role of religion in the early Greek Polis. Proceedings of the Third International Seminar on Ancient Greek Cult, organized by the Swedish Institute at Athens, 16-18 October 1992 (1996) 75-95; Krauter, Bürgerrecht (Anm. 9); Funke, „Fremde" (Anm. 9).

13 Vgl. dazu B. Smarczyk, Untersuchungen zur Religionspolitik und politischen Propaganda Athens im Delisch-Attischen Seebund (1990); dens., „Religion und Herrschaft: Der Delisch-Attische Seebunde“, Saeculum 58 (2007) 205-228.

14 Inscriptiones Graecae IX $1^{2}, 718$, bes. Zl. 1-4; s. auch Sylloge Inscriptionum Graecarum ${ }^{3}$ Nr. 47; R. Meiggs - D. M. Lewis, A Selection of Greek Historical Inscriptions to the End of the Fifth Century BC $\left(1988^{2}\right)$ Nr. 20; Körner, Gesetzestexte (Anm. 10) Nr. 49; H. van Effenterre - F. Ruzé, Nomima. Recueil d'inscriptions politiques et juridiques de l'archaïsme grec, Bd. 1 (1994) Nr. 43. 
auch in religiosis war, und wie sehr das Eingebundensein eines Bürgers in seine Polis gerade auch über die Kulte gefestigt werden sollte.

Die feste Verankerung der Religion im Gesamtsystem der Polis bedingte aber auch, dass die Zahl der verschiedenen Kulte und Gottheiten so unüberschaubar war wie die der griechischen Poleis und Stämme. Paul Cartledge hat einmal festgestellt, „that religion was above all the totality of public festivals celebrated by each of the hundreds of political communities; and the total was extraordinarily high, both absolutely and as proportion of the days in the year given over to festivals by the cities. Our evidence is unfortunately very incomplete and uneven. But altogether in excess of 300 public, state-run religious festivals are known to have been celebrated at over 250 places in honour of more than 400 deities. ${ }^{~} 15$

Diese Vielfalt religiöser und kultischer Erscheinungsformen ist aber nur die eine Seite der Medaille. Bei aller Diversifikation der religiösen Welt der Griechen, die sich auch in den Epiklesen der Götternamen in besonderer Weise widerspiegelt, ist es aber doch ein besonderes Charakteristikum, dass es - wie bereits eingangs vermerkt - quasi als eine Art Überbau auch die Vorstellung eines gemeinsamen religiösen Zusammenhaltes gab. ${ }^{16}$ Christiane SourvinouInwood hat davon gesprochen, dass „each polis was a religious system which formed part of the more complex world-of-the-polis system, interacting with the religious systems of the other poleis and with the Panhellenic religious dimension. " ${ }^{17}$ Mit den "panhellenischen Dimensionen" hat es allerdings seine besondere Bewandtnis, denn in gewisser Weise handelt es sich dabei um ein Konstrukt, was auch schon den antiken Zeitgenossen klar gewesen war. Schon die von Herodot geäußerte Auffassung über die Ursprünge des griechischen Pantheons weist in diese Richtung, wenn er sagt, dass erst Homer und Hesiod für die Hellenen „den Stammbaum der Götter geschaffen und den Göttern die

15 P. Cartledge, „The Greek Religious Festivals“, in: P. E. Easterling -J. V. Muir (Hgg.), Greek Religion and Society (1985) 98-99.

16 Die folgenden Ausführungen knüpfen unmittelbar an frühere Überlegungen an; vgl. dazu P. Funke, „Herodotus and the Major Sanctuaries of the Greek World“, in: V. Karagheorgis - J. Taifacos (Hgg.), The World of Herodotus. Proceedings of an International Conference held at the Foundation Anastasios G. Leventis, Nicosia, September 18-21, 2003 and organized by the Foundation Anastasios G. Leventis and the Faculty of Letters, University of Cyprus (2004) 159-167; dens., „Die Nabel der Welt“, in: T. Schmitt - W. Schmitz - A. Winterling (Hgg.), Gegenwärtige Antike - antike Gegenwarten. Kolloquium zum 60. Geburtstag von Rolf Rilinger (2005) 1-16; dens., „Fremde“ (Anm.9); dens., „Was ist der Griechen Vaterland? Einige Überlegungen zum Verhältnis von Raum und politischer Identität im antiken Griechenland“, Geographia Antiqua 14 (2005 / 2009) (im Druck).

17 Sourvinou-Inwood, „Polis Religion „(Anm. 4) 13. 
Beinamen gegeben und ihre Ämter und Fertigkeiten gesondert und ihre Gestalten deutlich gemacht haben". ${ }^{18}$

Dabei ist es für unsere Fragestellung unerheblich, inwieweit sich hinter dieser Äußerung ein religiöser Skeptizismus oder eine Distanzierung von einer Theogonie verbergen. Mir geht es nur um die bloße Feststellung, dass wir hier trotz aller Diversifikationen nicht nur in politicis, sondern auch in religiosis eine panhellenische Wahrnehmungsebene greifen, die unabhängig von der persönlichen Einstellung Herodots für das religiöse Denken der Griechen signifikant gewesen ist. Diese panhellenische Perspektive kam vor allem immer dann zum Tragen, wenn es um die Betonung der Zusammengehörigkeit und des $\mathrm{Zu}$ sammenhalts der Griechen ging. So konnte sich Aristagoras von Milet ebenso auf die $\theta \varepsilon o \grave{~ ' E \lambda \lambda \eta ́ v ı o ~ b e r u f e n ~ w i e ~ d e r ~ K o r i n t h e r ~ S o k l e e s ~ u n d ~ d e r ~ A t h e n e r ~}$ Hippias. ${ }^{19}$ Im Jahre 480 lehnten die Athener das persische Friedensangebot ab „aus Furcht vor dem Zeus der Hellenen“20; und Hegesistratos von Samos rief 479 in Delos die Griechen im Namen der ,gemeinsamen Götter “21 zur Fortführung des Kampfes gegen die Perser auf. Und als die Athener im Winter 480/ 79 angesichts der persischen Bedrohung die Einheit der Griechen beschworen, beteuerten sie nachdrücklich, dass sie nicht zu Verrätern der gemeinsamen

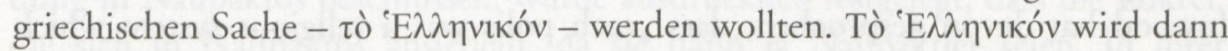
von den Athenern näher bestimmt. Neben dem gleichen Blut und der gemeinsamen Sprache werden vor allem die gemeinsamen Heiligtümer und Opfer als gesamtgriechisches Merkmal hervorgehoben. ${ }^{22}$

Diese panhellenische Sicht auf die griechische Götterwelt war nun eine wichtige Voraussetzung dafür, dass sich auch so Etwas wie eine panhellenisch orientierte sacred landscape entwickeln konnte, deren Grundlagen zwar in einer polisorientierten religiösen Welt verankert waren, die aber doch einen eigenen polisübergreifenden, eben panhellenischen Charakter besaß. ${ }^{23}$ Die Theoí Hellénioi wurden in einer Welt von Heiligtümern verortet, deren geographische Koordinaten durch ein gemeinsames religiöses Selbstverständnis der Griechen bestimmt wurde. Es war eine spezifische kultische Oikumene, die nicht nur aus

18 Herodot 2,53,2; s. dazu W. Burkert, „Herodot über die Namen der Götter: Polytheismus als historisches Problem“, Museum Helveticum 42 (1985) 121-132; R. Thomas, Herodotus in Context: Ethnography, Science and the Art of Persuasion (2000) 216-217.

19 Aristagoras: Herodot 5,49,3; Soklees: Herodot 5,92 ; Hippias: Herodot 5,93,1.

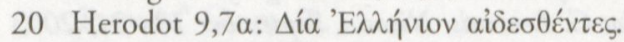

21 Herodot 9,90,2: $\theta$ coì kotvoí.

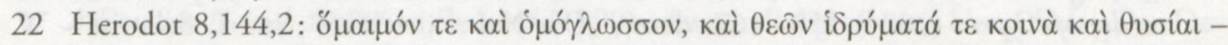
vgl. dazu Parker, Cleomenes (Anm. 8); D. Konstan, „To Hellenikon ethnos: Ethnicity and the Construction of Ancient Greek Identity", in: I. Malkin (Hg.), Ancient Perceptions of Greek Ethnicity (2001) 39-50; J. Hall, Hellenicity. Between Ethnicity and Culture (2002) $189 \mathrm{ff}$; K. Zacharia, „Herodotus' Four Markers of Greek Identity“, in: dies., Hellenism: Culture, Identity, and Ethnicity from Antiquity to Modernity (2008), 21-36.

23 Vgl. zum Folgenden Funke, „Herodotus“ (Anm. 15); dens., "Nabel“ (Anm. 15). 
der einfachen Summe einer Vielzahl von Tempeln und Kultplätzen bestand, sondern durch die Existenz einzelner, weit über einen engeren lokalen Bereich hinaus wirksamer Heiligtümer, die auch in der nichtgriechischen Welt in Ansehen standen, in besonderer Weise gekennzeichnet war.

Das Spezifische, das diese Welt zusammenhielt, das war eine panhellenische Akzeptanz und - als Kehrseite der Medaille - eine panhellenische Verfügbarkeit. Unbeschadet der administrativen Zuständigkeit einzelner Poleis, Ethne oder amphiktyonischer Verbände waren diese Heiligtümer in den Augen der Griechen in gewisser Weise panhellenisches Gemeingut. Es sei in diesem Zusammenhang nur auf die erste Klausel des Nikiasfriedens von 421 verwiesen, in der

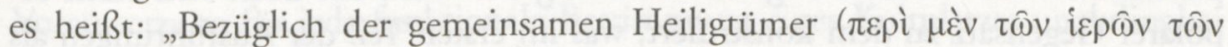

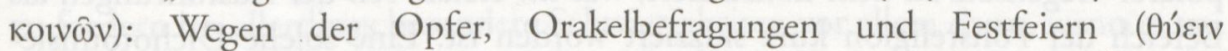

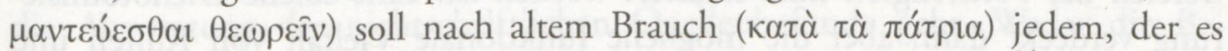
wolle, freier Zugang zu Wasser und zu Lande garantiert werden. "24 Auch wenn diese Regelung vor dem Hintergrund der damaligen athenisch - spartanischen Auseinandersetzungen um die Kontrolle Delphis gesehen werden muss, legt es

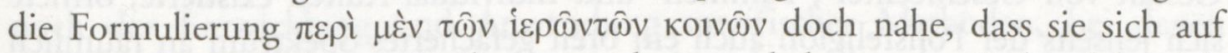
einen größeren Kreis von Heiligtümern bezogen haben muss. Es handelte sich hierbei ganz offensichtlich um nichts anderes als um eine Garantieerklärung für den besonderen Schutz der Heiligtümer, die nach allgemeiner Auffassung für alle Griechen von besonderer Bedeutung waren.

Dabei ist es bemerkenswert, dass es hier ebenso wie schon in der zitierten Rede der Athener keiner weiteren Erläuterungen bedurfte, um welche Heilig-

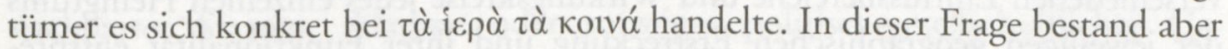
offenbar ein allseitiges Einverständnis, das für die griechischen Zeitgenossen

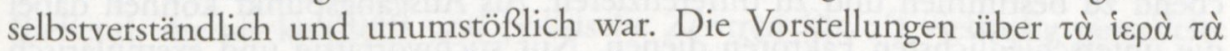
кoเvó im klassischen Griechenland beruhten also keineswegs nur auf einem eher unspezifischen emotionalen Empfinden, sondern waren sehr konkret mit bestimmten Heiligtümern verbunden, die in ihrer Gesamtheit eine sacred landscape formten und die als solche in einer mental map in den Köpfen der Griechen eingezeichnet waren. Hierbei handelte es sich unter anderem neben den letztlich keineswegs nur vier - Austragungsorten der großen panhellenischen Wettkämpfe vor allem um Orakel- und Heilkulte; aber auch Mysterienkulte wie die in Eleusis und Samothrake dürften dazu gehört haben - um nur einige Beispiele zu nennen.

Diese Heiligtümer, die aufs Ganze besehen die griechische Staatenwelt wie ein Netz umspannten und an allen Ecken und Enden miteinander verbanden, wurden durch ihre Kulte, Riten und Festfeiern zu Fixpunkten einer regelmäßigen Begegnung aller Hellenen und waren damit sogar weit mehr als bloße Elemente einer sacred landscape. Sie bildeten Orientierungsmarken einer pan-

24 Thukydides 5,18,2. 
hellenischen Landschaft, deren konkrete Grenzen eher unbestimmt waren und die - nicht zuletzt auch durch ein oft wucherndes Wachstum einer konstruierten panhellenischen syngeneia befördert - eine ständige Umgestaltung erfuhr. Es war ein imaginiertes Vaterland, dessen Bedeutung für die Selbstvergewisserung und den Zusammenhalt der eigentlich vaterlandslosen Hellenen nicht hoch genug eingeschätzt werden kann. ${ }^{25}$

In der Regel werden alle diese Heiligtümer unter dem Oberbegriff „panhellenisch" subsumiert $;{ }^{26}$ und die panhellenische Dimension kennzeichnet auch zweifellos einen spezifischen Charakterzug dieser Kultstätten. Mit einer solchen panhellenischen Fokussierung wird aber zugleich auch ein doch sehr stark bipolarer Gegensatz zu dem konstruiert, was im ersten Teil der Ausführungen als Bereich der Polisreligion kurz skizziert worden ist. Eine solche Dichotomisierung verdeckt dann aber die mögliche funktionale Vielfalt von Kulten und Heiligtümern - in religiosis wie in politicis. Denn so, wie innerhalb - oder auch unterhalb - der Polisreligion eine Vielzahl religiöser Erscheinungsformen in Gestalt von Geschlechter-, Familien- und Individual-Kulten existierte, öffnete sich jenseits der Polisreligion auch ein breit gefächertes Spektrum an räumlich wie auch funktional verschiedenen Einflussbereichen und Wirkungskreisen „polisübergreifender" Heiligtümer, das sich mit dem Begriff „panhellenisch“ nicht angemessen bezeichnen lässt. Stattdessen erscheint es mir angebrachter, in diesem Zusammenhang nicht vorschnell mit Sammelbegriffen wie "panhellenisch" oder "polisübergreifend“ zu operieren, sondern zunächst einmal die verschiedenen Einflussbereiche und Wirkungskreise jedes einzelnen Heiligtums der jeweiligen geographischen Erstreckung und ihrer Funktionalität entsprechend zu bestimmen und zu differenzieren. Als Ausgangspunkt können dabei die unterschiedlichsten Faktoren dienen. Nur stichwortartig und exemplarisch seinen hier als Kategorien genannt: die Herkunft und Typologie von Weihungen, die geographische Streuung von Asylieurkunden, die räumliche Erstreckung der Akzeptanz eines im Zusammenhang bestimmter Festfeiern verkündeten allgemeinen Landfriedens (ekecheiria), die Sitzverteilung in Amphiktyonenräten, der Einzugsbereich von Freilassungsurkunden, die Herkunft von Orakelanfragen. Mit Hilfe solcher Indikatoren sind ganz unterschiedlich dimensionierte, durchaus auch überlappende Einflussbereiche und Wirkungskreise auszumachen, die dann wiederum auch Rückschlüsse zulassen auf die jeweiligen Personengruppen und die Art ihrer Selbstidentifizierung mit dem entsprechenden Kult bzw. Kultplatz.

25 Vgl. dazu Funke, „Vaterland“ (Anm. 15).

26 S. etwa L. Briut Zaidman - P. Schmitt Pantel, Die Religion der Griechen. Kult und Mythos (1994) 112-140; zum Panhellenismus vgl. jetzt auch die umfassende Studie von L. G. Mitchell, Panhellenism and the barbarian in archaic and classical Greece (2007) (mit der älteren Literatur). 
Eine solche Vorgehensweise empfiehlt sich umso mehr, da durchaus ein und dasselbe Heiligtum sowohl auf lokaler und regionaler Ebene wie auch auf überregionaler und panhellenischer Ebene und noch darüber hinaus bis in nichtgriechische Bereiche hinein Wirksamkeit entfalten und Einfluss geltend machen konnte - aber eben nicht beliebig und nicht in jedem Fall. ${ }^{27}$ Funktionalität und räumliche Dimensionen scheinen vielmehr in einem sich gegenseitig bedingenden Wechselverhältnis zueinander zu stehen, dessen jeweilige Voraussetzungen und Rahmenbedingungen in jedem Einzelfall zu klären sind. Nur auf diese Weise lassen sich dann auch die jeweiligen politischen Dimensionen genauer bestimmen. Im Einzelfall birgt eine solche Analyse, die als Voraussetzung für jede funktionale Bestimmung eines Kultplatzes unabdingbar $\mathrm{zu}$ fordern ist, allerdings besondere Schwierigkeiten vor allem dann, wenn es um die Auswertung der nichtschriftlichen Quellenbefunde geht. Weder die Bestimmung des Objektes selbst noch die seiner Provenienz erlaubt zugleich auch schon sichere Rückschlüsse auf den funktionalen oder räumlichen Zuschnitt von Einflussbereichen und Wirkungskreisen eines Heiligtums. Hier bedarf es noch der Entwicklung und Anwendung spezifischer Methoden, bei denen qualitative und quantitative Analysen miteinander verbunden werden müssen, um zu entsprechenden Aussagen gelangen zu können.

Abschließend soll das im Vorangegangenen Skizzierte an einem Beispiel zumindest ansatzweise verdeutlicht werden: Einen spezifischen Typus polisübergreifender Heiligtümer stellten die so genannten amphiktyonischen Heiligtümer dar, die den kultischen Mittelpunkt von Zusammenschlüssen benachbarter Staaten, Stämme oder sonstiger politisch-gesellschaftlicher Gemeinschaften bildeten. ${ }^{28}$ Dabei ist der Übergang zu den Heiligtümern durchaus fließend, die als kultische und politische Zentren für oft vielstufig gestaffelte und vielfach auch nur sehr locker verbundene stammes- oder bundesstaatlich strukturierte Entitäten fungierten. Von den amphiktyonischen Verbänden ist die delphische Amphiktyonie zweifellos der bekannteste, aber keineswegs der ein-

$27 \mathrm{Zu}$ welchen Erträgen einer solcher Ansatz führen kann, hat die exemplarische Untersuchung von N. Moustakis, Heiligtümer als politische Zentren: Untersuchungen zu den multidimensionalen Wirkungsgebieten von polisübergreifenden Heiligtümern im antiken Epirus (2006) gezeigt.

28 Vgl. hierzu die entsprechenden Ausführungen bei K. Tausend, Amphiktyonie und Symmachie: Formen zwischenstaatlicher Beziehungen im archaischen Griechenland (1992); E. Baltrusch, Symmachie und Spondai: Untersuchungen zum griechischen Völkerrecht der archaischen und klassischen Zeit (8. - 5. Jahrhundert v. Chr.), (1994); P. Siewert - L. Aigner-Foresti (Hgg.), Föderalismus in der griechischen und römischen Antike (2005), 1924; P. Funke, „Alte Grenzen - neue Grenzen: Formen polisübergreifender Machtbildung in klassischer und hellenistischer Zeit", in: R. Albertz - P. Funke (Hgg.), Räume und Grenze: Topologische Konzepte in den antiken Kulturen des östlichen Mittelmeerraumes (2007) 187-204; E. Baltrusch, Außenpolitik, Bünde und Reichsbildung in der Antike (2008). 
zige Fall in der griechischen Staatenwelt. ${ }^{29}$ Das delphische Heiligtum fungierte nun aber nicht nur als amphiktyonischer, also regional begrenzter Kultort, der in Mittelgriechenland stammes- und polisübergreifend eine auch politisch wirksame integrative Kraft darstellte; vielmehr war Delphi zu bestimmten Zeiten auch ein kultisches Zentrum panhellenischer Wettkämpfe und eine sogar weit über den griechischen Raum hinaus wirksame Orakelstätte und blieb zugleich doch immer auch zentraler Ort einer lokal begrenzten Polisreligion.

Der Blick soll hier aber ausschließlich auf den Aspekt der Amphiktyonie gerichtet bleiben. Die Bedeutung amphiktyonischer Staatenbünden für die Genese und Ausgestaltung spezifischer zwischenstaatlicher, aber auch eigenstaatlicher Strukturen in der Antike wird in der jüngeren Forschung weit unterschätzt und aufgrund einer einseitigen Fixierung auf den jeweiligen kultischen Charakter kaum noch wahrgenommen. Hier scheint das Gespür für die unmittelbare Wechselwirkung und den untrennbaren Zusammenhang von Religion und Kult einerseits und dem, was wir „Staatlichkeit“ nennen könnten, aus dem Blick geraten zu sein. Es ist erstaunlich, wie stark in der neueren Forschung die politischen Aspekte der Amphiktyonien gegenüber den rein sakralen zurückgetreten sind. Es muss daher darum gehen, das Augenmerk wieder verstärkt auf die multifunktionale Organisationsstruktur von Amphiktyonien und auch anderen überregionalen Heiligtümern und Kultverbänden zu richten und in diesem Zusammenhang insbesondere auch die Regelungen und Mechanismen der Binnenstrukturen dieser zwischenstaatlichen Verbünde mit zu berücksichtigen. Lassen sich hier doch überaus interessante Ansätze finden, zumindest Grundregeln eines friedlichen und geordneten Zusammenlebens eigenständiger, zunächst meist unmittelbar benachbarter, aber keineswegs zwingend ethnisch verwandter Staaten festzulegen, die durch religiöse Rückbindung abgesichert wurden und deren Bruch durchaus sanktioniert werden konnte. ${ }^{30}$

29 Vgl. G. Busolt - H. Swoboda, Griechische Staatskunde, Bd. $2\left(1926^{3}\right) 1280-1310$ sowie die in Anm. 27 aufgeführte Literatur; s. auch J. Mylonopoulos, „Von Helike nach Tainaron und von Kalauria nach Samikon: Amphiktyonische Heiligtümer des Poseidon auf der Peloponnes", in: K. Freitag - P. Funke - M. Haake (Hgg.), Kult - Politik Ethnos: Überregionale Heiligtümer im Spannungsfeld von Kult und Politik (2006) 121 167; speziell zur pyläisch-delphischen Amphiktyonie s. F. Lefevre, L'amphictionie pyléodelphique: histoire et institutions (1998); P. Sánchez, P., Lamphictionie des Pyles et de Delphes: Recherches sur son rôle historique, des origines au IIe siècle de notre ère (2001).

30 Die für die delphisch-pyläische Amphiktyonie belegten Regeln sprechen dafür, dass auch die Mitglieder anderer Amphiktyonien, die politisch unabhängige Staaten blieben, sich in durch Eide bekräftigten Übereinkünften verpflichteten, auch im Konfliktfall bestimmte Regeln im zwischenstaatlichen Miteinander einzuhalten, um wenigstens die Existenzgrundlagen aller Amphiktyonen sicherzustellen. Somit fungierten die griechischen Amphiktyonien als eine sehr frühe Instanz zur Stabilisierung zwischenstaatlicher Beziehungen. - Zum delphischen Eid vgl. Aischines, de falsa legatione (2) 115. Selbst wenn die bei Aischines überlieferte Eidesformel ein spätes Konstrukt sein sollte [so etwa 
So besehen erhalten amphiktyonische Kultzentren einen Bezugsrahmen und einen Funktionszusammenhang, die über die rein religiösen und sakralrechtlichen Sphären weit hinausreichen. Auf diese Weise konnten überregionale Heiligtümer auch in politicis eine große Integrationskraft entfalten und identitätsstiftend bzw. -sichernd wirken für diejenigen, die Teilhaber dieser Heiligtümer waren. Kultgemeinschaft und politische Gemeinschaft waren dabei aufs engste miteinander verwoben, jedoch dergestalt, dass die amphiktyonische Identität sich zur je eigenstaatlichen Identität nicht konkurrierend, sondern ergänzend verhielt. Die Relationen zwischen diesen Identitäten waren allerdings nicht starr, sondern konnten sich vielmehr in ganz unterschiedliche Richtungen entwickeln: Einerseits konnte das Zusammenwirken an den überregionalen Kulten und Heiligtümern für die beteiligten Gemeinschaften zu einer Stabilisierung auch der je eigenen „staatlichen“ oder ethnischen Identität beitragen, indem ganz bewusst der übergreifende amphiktyonische bzw. zwischenstaatliche Charakter auch in der institutionellen Ausgestaltung der Kultverwaltung und -praxis etwa in Form eines repräsentativ zusammengesetzten Amphiktyonenrates zum Tragen kam; andererseits kam es aber durchaus auch zu sehr weitgehenden Annäherungen zwischen den am Kult beteiligten Gemeinschaften auch in politicis immer da, wo staaten- und stammesbündische Amphiktyonien bundesstaatliche Zusammenschlüsse generierten und die überregionalen Kultzentren zum Kulminationspunkt dieser Entwicklung wurden, um vor allem auch mögliche politische Spannungen zwischen den einzelnen Gliedstaaten eines Bundes aufzufangen und zu kompensieren. ${ }^{31}$

Auch wenn dieser sehr knappe Verweis auf die griechischen Amphiktyonien nur auf einen einzelnen Aspekt aus der Fülle möglicher Einflussbereiche und Wirkungskreise Bezug genommen hat, mag dennoch deutlich geworden sein, wie sich durch eine entsprechende Differenzierung Vielfalt und Komplexität nicht nur, aber auch der politischen Funktionen von Heiligtümern besser erschließen lassen. Entscheidende Orientierungspunkte waren offenbar stets Identitätsstiftung bzw. Identitätssicherung durch Integration und Abgrenzung im Kultischen als Ausdruck einer eben auch politischen Selbstvergewisserung.

P. Sánchez, „Le serment amphictionique (Aeschin. Legat. [2] 115): un faux de $I^{\mathrm{e}}$ siècle?", Historia 46 (1997) 158-171; s. aber auch Lefevre, L'amphictionie (Anm. 28) $147-151]$, zeigt doch die diesem Konstrukt dann zugrunde liegende Sichtweise, dass die politische Funktion der delphischen Amphiktyonie als Regulativ im zwischenstaatlichen Miteinander erkannt und im Blick war.

31 Vgl. die für diesen Sachverhalt bezeichnende Notiz bei Polybios 2,39,5-6, der zufolge sich die süditalienischen Städte Kroton, Sybaris und Kaulonia wohl um die Mitte des 5. Jhs. v. Chr. zu einem Städtebund zusammengeschlossen und ein gemeinsames Heiligtum für Zeus Homarios gegründet hatten, das als Versammlungsort für die Bundesversammlung und den Bundesrat diente. Vgl. dazu F. W. Walbank, „Hellenes and Achaians: ,Greek Nationality' Revisited“, in: P. Flensted-Jensen (Hg.), Further Studies in the Ancient Greek Polis (2000) 23-24. 